\title{
Supresión de Modos de Vibración Acústicos con un Resonador Helmholtz
}

\author{
Andrés Guiguet e Reinaldo Welti \\ Facultad de Ciencias Exactas, Ingeniería y Agrimensura, \\ Universidad Nacional de Rosario, Avda. Pellegrini 250, (2000) Rosario, Argentina
}

Recebido em 01 de abril, 2003. Aceito em 09 de junho, 2003.

\begin{abstract}
La inserción de un Resonador Helmholtz $(R H)$ en las paredes laterales de un tubo, con ondas estacionarias en su interior, logra suprimir uno o más de sus modos resonantes si se elige adecuadamente la frecuencia del resonador. El $R H$ puede actuar también como filtro de ondas propagantes. En este caso, el $R H$ atenúa las ondas en un rango de frecuencia muy selectivo. En la mayoría de los textos de acústica, solamente se desarrolla la teoría que explica el filtrado de ondas propagantes. Sin embargo, en los laboratorios de física básica, donde se dispone solamente de tubos de Kundt de pequeña longitud, no es simple realizar un arreglo experimental que asegure la presencia de ondas propagantes puras en su interior. La falta de una teoría para ondas estacionarias y las dificultades experimentales que señalamos han producido algunas confusiones en trabajos que tratan sobre el tema. En este artículo se presenta un modelo teórico que describe satisfactoriamente el comportamiento del $R H$ cuando funciona como filtro de ondas estacionarias y se marcan las diferencias con la situación en que opera como filtro de ondas propagantes.
\end{abstract}

The insertion of a Helmholtz resonator (HR) in the lateral walls of a tube containing stationary waves in its interior can be used to suppress one or more resonant modes if one chooses the resonator frequency appropriately. The HR can also operates like a filter for traveling waves. In this case, the HR attenuates the waves in a very selective frequency range. In textbooks on Acoustics, only the filtering process of traveling waves is explained. However, it is not simple to carry out experimental arrangement on small length Kundt tubes available in introductory physics laboratories that assures the presence of pure traveling waves inside the tube. Since there is not a theory for stationary waves and the experimental difficulties have produced some confusion in works approaching this topic, we present here a theoretical model to explain the RH behavior when it works like a filter for stationary waves and we point out the differences when it operates like a traveling waves filter.

\section{Introducción}

Como la cantidad de ruido ambiente ha estado creciendo en los últimos años, tanto en su variedad como en su nivel, se ha producido una toma de conciencia también creciente acerca del problema de la contaminación sonora. Surge, por lo tanto, la necesidad de eliminar estos ruidos indeseables. Los filtros acústicos son dispositivos que tienen la propiedad de atenuar o suprimir un cierto ancho de banda de frecuencias del sonido o ruido indeseable. Son ejemplos de filtros acústicos, el caño de escape de un automóvil, los silenciadores de las armas de fuego, etc.

Cameron y Russell (1996), plantean una serie de excelentes trabajos de laboratorio que tienen como objetivo introducir a los estudiantes en la ingeniería del sonido y la vibración. Uno de estos trabajos está dedicado al estudio y la construcción de diversos filtros acústicos. En particular proponen la utilización de un Resonador Helmholtz (RH) como filtro para absorber vibraciones de bajas frecuencias en un rango de frecuencia muy selectivo. En efecto, los cálculos teóricos de Kinsler et al. (1982) muestran que, en esta situación, se produce una fuerte atenuación de la onda propa- gante en un rango de frecuencias que se extiende hasta una octava sobre ambos lados de la frecuencia de resonancia del RH.

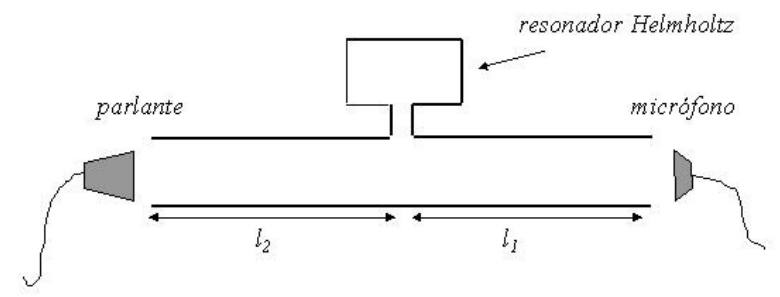

Figura 1. Resonador Helmholtz insertado lateralmente en un tubo abierto en ambos extremos.

El dispositivo que Russell propone para hacer este experimento (ver figura 1) es un tubo de longitud $L$, abierto en sus dos extremos y con un parlante en su extremo izquierdo, que vibra con amplitud constante y frecuencia variable. En estas condiciones, sin embargo, no es posible crear una onda propagante en el interior del tubo, pues la interferencia entre la onda que viaja de izquierda a derecha y la onda reflejada en el extremo derecho dan como resultado ondas estacionarias. Para determinadas frecuencias, denominadas frecu- 
encias de resonancia o frecuencias naturales, se forman en el interior del tubo, patrones de ondas estacionarias (modos normales), de amplitudes relativamente grandes. La teoría desarrollada por Kinsler et al. no es, por lo tanto, aplicable al experimento planeado de esta manera. Es interesante comentar que Brewer (1992), quien trabajó con un diseño similar al de Russell, concluye que los resultados de su experimento "no se corresponden con los resultados predichos en los textos estándares de acústica". Esto ocurre porque la teoría que se desarrolla en esos textos es para ondas propagantes y no para ondas estacionarias con las que él trabaja.

La inserción de un $R H$ en las paredes laterales de un tubo abierto en sus dos extremos produce una modificación de sus modos normales y de sus respectivas frecuencias naturales de oscilación. En este trabajo calculamos teóricamente la amplitud de respuesta del tubo en función de la frecuencia de excitación. Mostramos que la inserción del $R H$ produce una modificación importante de la amplitud de respuesta solamente en un pequeño rango de frecuencias alrededor de la frecuencia de resonancia del $R H$ y que si esta frecuencia se elige adecuadamente se pueden suprimir uno o dos modos resonantes. Estos resultados teóricos concuerdan muy bien con los resultados experimentales que obtuvimos con un dispositivo experimental similar al que se muestra en la figura 1. En particular, con esta teoría se explican los resultados experimentales de Russell y de Brewer.

En la sección 2 se deduce la expresión para la admitancia de entrada de un tubo de longitud $L$, abierto en sus dos extremos, al que se le inserta lateralmente un $R H$. En la sección 3 se presentan los resultados experimentales y se comparan con los valores que predice la teoría desarrollada en la sección 2. En la sección 4 se analiza la teoría estándar del filtro $R H$ para ondas propagantes y se señalan las diferencias con el caso en que se tengan ondas estacionarias. En el Anexo 1 se explica brevemente el funcionamiento de un $R H$ y se definen los parámetros acústicos relevantes de este dispositivo. En el Anexo 2 se describen el diseño experimental y el material que hemos utilizado.

\section{Efecto de un RH sobre la amplitud de respuesta de un tubo abierto en sus dos extremos}

En esta sección vamos a deducir la expresión para la amplitud de respuesta en el dominio de la frecuencia de un tubo abierto en sus dos extremos. La comparación de las amplitudes de respuesta del tubo, con o sin el $R H$, permite cuantificar el comportamiento del $R H$ como filtro.

La amplitud de respuesta del tubo es directamente proporcional a la admitancia de entrada del tubo (la inversa de la impedancia de entrada). Para calcular la impedancia de entrada del tubo es conveniente convertir al sistema de la figura 1 en un sistema eléctrico análogo en el cual el movimiento del fluido es equivalente a la corriente en una línea de transmisión eléctrica. El análogo eléctrico de la presión $p$ es la tensión, y un análogo acústico de la corriente es el flujo de volumen $U=S u$, donde $u$ es la velocidad del fluido y $S$ la sección del tubo.La impedancia acústica del tubo $Z_{0}$, (el equivalente a la impedancia característica de una línea de transmisión eléctrica), es el cociente entre la presión acústica $p$ y el flujo de volumen $U, Z_{0}=p / U$.

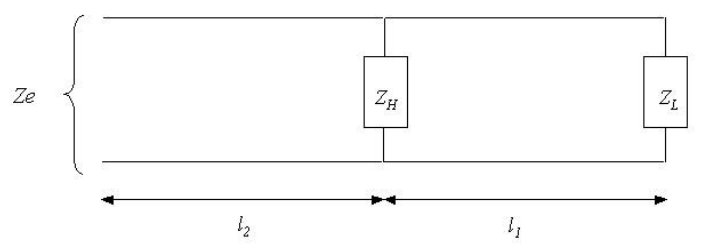

Figura 2. Circuito eléctrico equivalente del sistema acústico de la figura 1.

La impedancia de una porción de un sistema acústico, de dimensiones pequeñas comparadas con la longitud de onda, (el equivalente a un elemento de parámetros concentrados eléctrico), es el cociente entre la diferencia de presión $p$ que se está ejerciendo sobre este elemento y el flujo de volumen entrante al mismo $U$. El resonador Helmholtz es un sistema acústico de pequeña dimensión comparada con la longitud de onda. En el Anexo 1 se calcula su impedancia acústica $Z_{H}$. En el extremo del tubo, que está abierto a la atmósfera, la presión acústica es siempre cero. Puede, por lo tanto, considerarse como un "corto circuito", esto es, como un elemento de impedancia acústica cero.

En la figura 2 se muestra el circuito eléctrico equivalente del dispositivo que se muestra en la figura 1. Para calcular la impedancia de entrada de este sistema debemos calcular la impedancia en paralelo $Z_{p}$ entre la impedancia $Z_{1}$ de la porción del tubo de longitud $l_{1}$ que termina en una impedancia acústica $Z_{L}$ y el resonador Helmholtz que tiene una impedancia $Z_{H}$. Finalmente la impedancia de entrada $Z$ es la impedancia de un tubo de longitud $l_{2}$ que termina en una impedancia $Z_{p}$.

La impedancia $Z_{1}$ del tubo de longitud $l_{1}$ que termina en la impedancia $Z_{L}$ es

$$
Z_{1}=Z_{0} \frac{\frac{Z_{L}}{Z_{0}}+j \tan k l_{1}}{1+j \frac{Z_{L}}{Z_{0}} \tan k l_{1}}
$$

donde $Z_{0}=\rho_{o} c / S$ es la impedancia acústica del tubo, $\rho_{o}$ la densidad del aire no perturbado, $c$ la velocidad del sonido, $k=2 \pi / \lambda$ el número de onda y $\lambda$ la longitud de onda.

La impedancia $Z_{e}$ del paralelo entre la impedancia $Z_{1}$ y la impedancia del resonador $Z_{H}$ es

$$
Z_{p}=\frac{Z_{1} Z_{H}}{Z_{1}+Z_{H}}
$$

La impedancia de entrada $Z_{e}$ es la impedancia de la porción del tubo de longitud $l_{2}$ que termina en la impedancia $Z_{p}$, esto es:

$$
Z_{e}=Z_{0} \frac{\frac{Z_{p}}{Z_{0}}+j \tan k l_{2}}{1+j \frac{Z_{p}}{Z_{0}} \tan k l_{2}}
$$

Si reemplazamos (2) en (3) obtenemos:

$$
Z_{1}=Z_{0} \frac{\frac{Z_{1}}{Z_{0}}+j \tan k l_{2}+j \frac{Z_{1}}{Z_{H}} \tan k l_{2}}{1+j \frac{Z_{1}}{Z_{0}} \tan k l_{2}+\frac{Z_{1}}{Z_{H}}}
$$


Como el extremo derecho del tubo está abierto $Z_{L}=0$, entonces:

$$
Z_{1}=j Z_{0} \tan k l_{1}
$$

Reemplazando (5) en (4) obtenemos

$$
Z_{e}=Z_{0} \frac{j \tan k l_{1}+j \tan k l_{2}-\frac{Z_{0}}{Z_{H}} \tan k l_{1} \tan k l_{2}}{1-\tan k l_{1} \tan k l_{2}+j \frac{Z_{0}}{Z_{H}} \tan k l_{1}}
$$

Trabajando algebraicamente y utilizando identidades trigonométricas se obtiene:

$$
Z_{e}=j Z_{0} \frac{\sin k L+j \frac{Z_{0}}{Z_{H}} \sin k l_{1} \sin k l_{2}}{\cos k L+j \frac{Z_{0}}{Z_{H}} \sin k l_{1} \sin k l_{2}}
$$

Si se retira el $R H$ de las paredes del tubo, $Z_{H} \rightarrow \infty, \mathrm{y}$ la ecuación (7) se reduce a la impedancia de entrada de un tubo abierto de longitud $L$.

Si descomponemos la impedancia acústica del resonador en su parte resistiva y reactiva $Z_{H}=R_{H}+j X_{H}$, obtenemos

$$
Z_{e}=j Z_{0} \frac{X_{H} \sin k L+Z_{0} \sin k l_{1} \sin k l_{2}-j R_{H} \sin k L}{X_{H} \cos k L+Z_{0} \sin k l_{1} \cos k l_{2}-j R_{H} \cos k L}
$$

Finalmente, el módulo de la admitancia de entrada $Y_{e}$ es

$$
\left|Y_{e}\right|=\frac{1}{\left|Z_{e}\right|}=\frac{1}{Z_{0}} \sqrt{\frac{\left(x_{H} \cos k L+\sin k l_{1} \cos k l_{2}\right)^{2}+\left(r_{H} \cos k L\right)^{2}}{\left(x_{H} \sin k L+\sin k l_{1} \sin k l_{2}\right)^{2}+\left(r_{H} \sin k L\right)^{2}}}
$$

donde

$$
x_{H}=\frac{X_{H}}{Z_{0}}=\varkappa\left(\frac{f}{f_{H}}-\frac{f_{H}}{f}\right) \quad y r_{H}=\frac{R_{H}}{Z_{0}}
$$

En (10), $\varkappa$ es una constante que depende solamente de los parámetros del $R H$ y de la sección del tubo (ver Anexo 1).

Si la impedancia del parlante es pequeña, el parlante entrega la máxima potencia en las frecuencias para las cuales la admitancia de entrada del tubo es máxima. Estas frecuencias son las frecuencias de resonancia del sistema. En la próxima sección vamos a comparar la amplitud de respuesta medida con la que se calcula mediante la ecuación (9).

\section{Resultados experimentales. Su- presión de modos normales}

Si la frecuencia del parlante de la figura 1, coincide con la frecuencia de uno de los modos de oscilación del sistema tubo $+R H$, se crea una onda de relativamente gran amplitud. La gráfica de estas amplitudes en función de la frecuencia es la amplitud de respuesta del sistema.

En la figura 3 se muestra, mediante líneas llenas que unen los valores medidos (triángulos), la amplitud de respuesta del sistema $t u b o+R H$, y mediante líneas punteadas que unen los valores medidos (círculos) la amplitud de respuesta del tubo solo. La frecuencia del resonador $(342 \mathrm{~Hz})$ que se utilizó es muy próxima a la de la segunda resonancia del tubo ( $340 \mathrm{~Hz}$ ). Observemos que la inserción del este $R H$ suprime esta resonancia, mientras que la frecuencia de la primera resonancia $(170 \mathrm{~Hz})$ se corre hacia la izquierda en aproximadamente $10 \mathrm{~Hz}$. La tercera resonancia (no mostrada en la figura) se corre en la misma cantidad hacia la derecha. Tanto la primera como la tercera resonancia disminuyen su amplitud en un factor $0.6(-5 d B)$. Un hecho notable es la aparición de dos pequeños picos, en 295 y 400 $H z$, a uno y otro lado de la frecuencia del resonador.

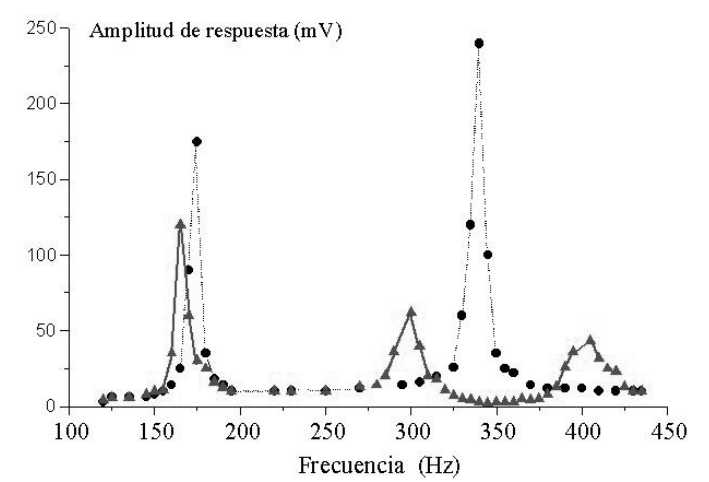

Figura 3. Medidas de la amplitud de respuesta del tubo en función de la frecuencia. En líneas punteadas (uniendo círculos) sin el resonador y en líneas llenas (uniendo triángulos) con el resonador insertado.

La curva de la figura 4, en línea punteada, muestra las amplitud de respuesta teórica del tubo sin el resonador y la curva en línea llena, la amplitud de respuesta del sistema tubo $+R H$ que se calcula mediante la ecuación (9). Se tomó para $r_{H}$ un valor igual a 0.1 por dos motivos: uno porque la curva que se obtiene de esta manera es la que mejor se ajusta a los resultados experimentales, y el otro porque éste es el valor que hemos determinado experimentalmente cuando medimos el coeficiente de transmisión (ver sección 5). La curva teórica refleja satisfactoriamente el comportamiento de la curva experimental. Se observa, en la figura 4, que los resultados teóricos predicen que la segunda resonancia desaparece, que la primera resonancia disminuye su amplitud y se corre hacia la izquierda y que aparecen dos pequeños picos a uno y otro lado de la segunda resonancia. 
En los cálculos teóricos estos dos picos están corridos aproximadamente a $\pm 80 \mathrm{~Hz}$ de la frecuencia del resonador (los valores medidos para estos corrimientos son de $\pm 60 \mathrm{~Hz}$ y la frecuencia de la primera resonancia se corre $20 \mathrm{~Hz}$ hacia la izquierda (el valor medido es de $10 \mathrm{~Hz}$ ).

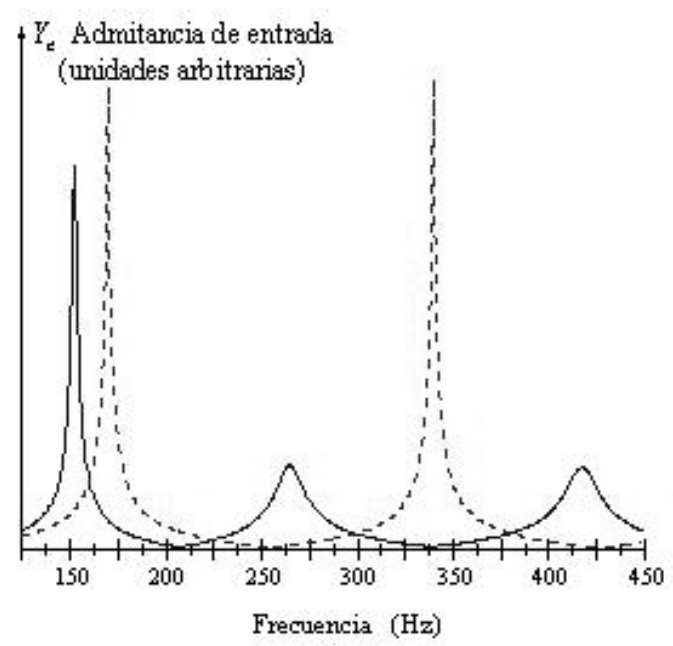

Figura 4. Valores teóricos de la amplitud de respuesta del tubo en función de la frecuencia. En líneas punteadas sin el resonador y en líneas llenas con el resonador.

Los cálculos teóricos permiten confirmar que los dos pequeños picos en 295 y $400 \mathrm{~Hz}$, respectivamente, son frecuencias de resonancia del sistema tubo $+R H$. En efecto, si hacemos $r_{H}=0$ la admitancia teórica resulta infinita en estas frecuencias. Si la resistencia del resonador es muy pequeña $\left(r_{H} \leqslant 0.01\right)$, estas resonancias tienen amplitudes muy grandes y el "filtro" elimina el pico resonante que coincide con su frecuencia $f_{H}$, pero introduce otros dos, lo que podría significar en ciertas situaciones un comportamiento no deseado.

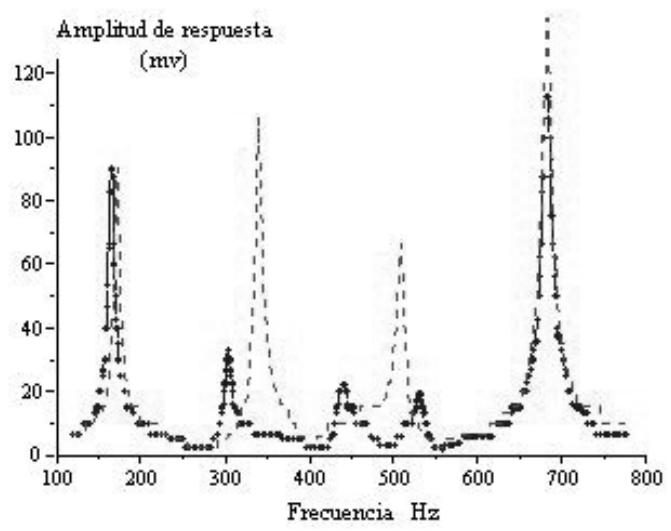

Figura 5. Valores medidos de la amplitud de respuesta del tubo en función de la frecuencia. En líneas de puntos chicos sin el resonador, y en líneas de puntos grandes con un resonador de 410 $H z$.

En la figura 5 se muestra, en líneas de puntos grandes, la amplitud de respuesta en función de la frecuencia cuando se inserta en el tubo un resonador de frecuencia $f_{H}=410 \mathrm{~Hz}$ (resonador $B$ ). En esta situación se observa que la segunda resonancia desaparece totalmente mientras que la tercera, de
$510 \mathrm{~Hz}$, se corre hacia la derecha y su amplitud disminuye fuertemente. La primera resonancia se corre levemente hacia la izquierda y la quinta queda prácticamente sin variaciones en su amplitud y frecuencia. Se observa también la aparición de dos pequeños picos en $300 \mathrm{~Hz}$ y $430 \mathrm{~Hz}$.

La curva en líneas llenas de la figura 6 es la gráfica de la admitancia de entrada del sistema compuesto por el tubo y el $R H$ que se calcula con la ecuación (9) de la sección 3, para un resonador cuyos parámetros son: $f_{H}=410 \mathrm{~Hz}, \varkappa$ $=1,2$. Para el cálculo teórico se supuso también que $r_{H}=$ 0,1 . Obtenemos otra vez, un buen acuerdo entre las curvas teóricas (figura 6) y las experimentales (figura 5).

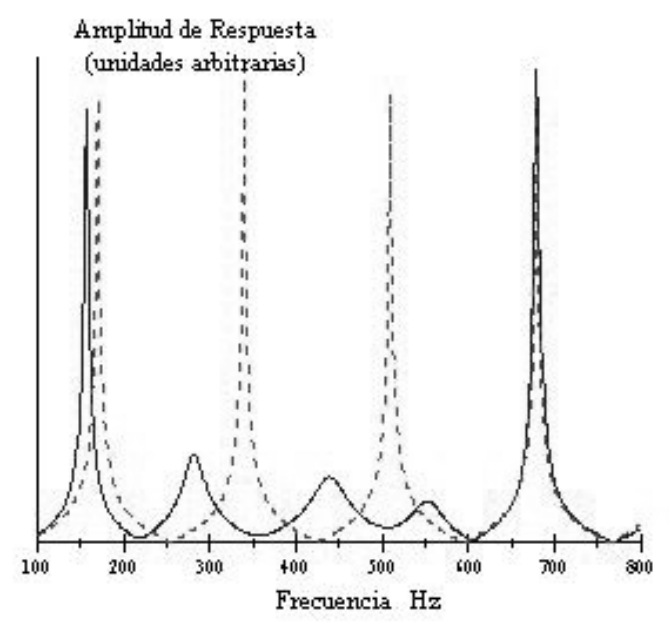

Figura 6. Curvas teóricas de la amplitud de respuesta en función de la frecuencia. En líeas punteadas sin el resonador y en líneas llenas con un resonador de $420 \mathrm{~Hz}$.

\section{Efecto de un RH sobre las ondas propagantes}

En la figura 7 se muestra esquemáticamente a un $R H$ insertado en las paredes laterales de un tubo de longitud semiinfinita. En esta figura, $A$ es la amplitud de una onda de frecuencia $f$, que viaja de izquierda a derecha hacia el punto de inserción, mientras que $B$ y $C$ son la amplitudes de la onda reflejada y transmitida, en ese punto, respectivamente.

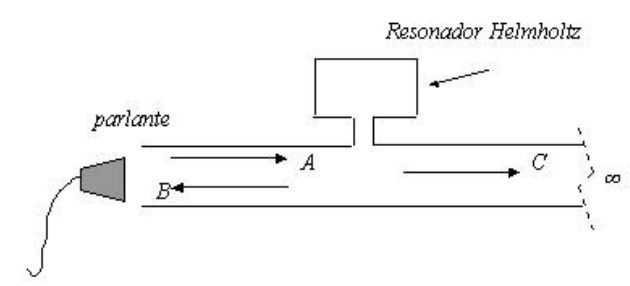

Figura 7. Resonador Helmholtz insertado en un tubo semiinfinito.

La razón entre la potencia transmitida y la potencia incidente es el coeficiente de transmisión de potencia (Kinsler et al, 1982) que viene dado por: 


$$
T=\left(\frac{C}{A}\right)^{2}=\frac{r_{H}^{2}+x_{H}^{2}}{\left(1 / 2+r_{H}^{2}\right)+x_{H}^{2}}
$$

Como en nuestra experiencia se cumple que $r_{H} \leqslant 0,1$ vamos a despreciar $r_{H}$ en el denominador de la ecuación (11) pero lo mantendremos en el numerador, para no tener un valor cero del coeficiente de transmisión para la frecuencia que anula la reactancia (que es la frecuencia de resonancia del resonador).

Si reemplazamos (10) en (11) se obtiene la siguiente expresión para el coeficiente de transmisión:

$$
T=\frac{r_{H}^{2}+\varkappa^{2}\left(f / f_{H}-f_{H} / f\right)^{2}}{1 / 4+\varkappa^{2}\left(f / f_{H}-f_{H} / f\right)^{2}}
$$

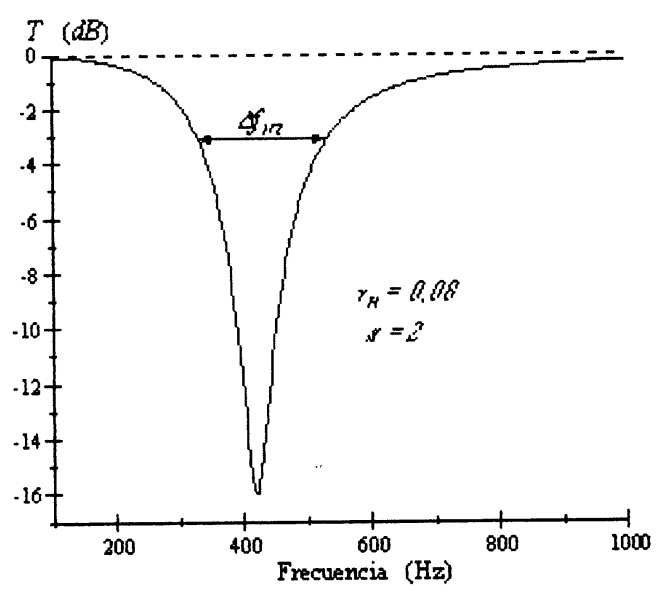

Figura 8. Curva teórica para el coeficiente de transmisión.

En la figura 8 se muestra una gráfica de $T$ en función de $f$ para los siguientes valores, $r_{H}=0,08, \varkappa=2 \mathrm{y}$ $f_{H}=410 \mathrm{~Hz}$.

El mínimo de $T$, que se obtiene para $f=f_{H}$, es igual a $4 r_{H}^{2}$. Si $r_{H} \ll 1$, el ancho de banda en frecuencia $\Delta f_{1 / 2}$ del filtro, definido como el intervalo de frecuencias para el cual $T \leq 1 / 2$, viene dado por

$$
\Delta f_{1 / 2} \approx \frac{f_{H}}{2 \varkappa}
$$

\section{Resultados experimentales. Coe- ficiente de transmisión para ondas propagantes}

El dispositivo experimental que se utilizó es similar al que se muestra en la figura 7. Para que en el interior del tubo se tengan solamente ondas propagantes (y no ondas estacionarias) se debe colocar un absorbente del sonido en el extremo derecho del tubo. El absorbente que hemos utilizado es de lana de vidrio compacta de $30 \mathrm{~cm}$ de longitud; comienza con un diámetro igual al del tubo y gradualmente va disminuyendo (como en un cono) en la dirección en que se encuentra el parlante. La efectividad de esta terminación absorbente se comprobó midiendo la amplitud de respuesta cuando el tubo (sin la inserción del $R H$ ) era excitado con una señal de amplitud constante y frecuencia variable. La amplitud de respuesta se mantuvo relativamente independiente de la frecuencia en el intervalo 300 a $1400 \mathrm{~Hz}$. Sus variaciones, sin embargo, aún cuando se mantenían menores que $\pm 3 d B$, indicaban la existencia de ondas reflejadas. Cameron y Brewer no utilizaron una terminación absorbente $y$, por lo tanto, en su experiencia se tienen ondas estacionarias y no propagantes. Este es el motivo por el cual tienen dificultades para analizar sus resultados mediante el coeficiente de transmisión para ondas propagantes.

En las figuras 9 y 10 se muestran, respectivamente, los coeficientes de transmisión que se midieron utilizando el resonador $\mathrm{C}$ de frecuencia $f_{H}=720 \pm 10 \mathrm{~Hz}$ y el resonador $B$ de frecuencia $f_{H}=410 \pm 10 H z$.

Las curvas de transmisión medidas coinciden satisfactoriamente con las curvas teóricas que se muestran en estas figuras en líneas finas punteadas. Las amplitudes transmitidas muestran, como era de esperar, una fuerte disminución en el entorno de la frecuencia del resonador. Las curvas experimentales de la figura 9 y 10 se desvían apreciablemente de la curva teórica para frecuencias que están entre 450 y $550 \mathrm{~Hz}$. En este intervalo las curvas experimentales muestran un pequeño máximo relativo. Este comportamiento de la amplitud medida puede ser ocasionado por la existencia de ondas estacionarias en el interior del tubo que se originan por el deficiente funcionamiento de la terminación absorbente. El dispositivo de forma cónica y de lana de vidrio que se utiliza como terminación "perfecta" debe tener, para su mejor funcionamiento, una longitud mayor que la mayor longitud de onda utilizada (que en este trabajo es del orden de $1 m$ ). La terminación que utilizamos, sin embargo, no supera los $0,30 \mathrm{~cm}$ debido a la pequeña longitud $(1 \mathrm{~m})$ del tubo de Kundt que utilizamos.

En la figura 10 se observa que el mínimo valor de $T$ para el resonador $B$ es de aproximadamente $-13 d B$. De acuerdo con la teoría de la sección 3 , el valor mínimo de $T$ es $4 r_{H}^{2}$. A partir de este valor medido concluimos que $r_{H}=R_{H} / Z_{0} \approx 0.11$ para este $R H$.

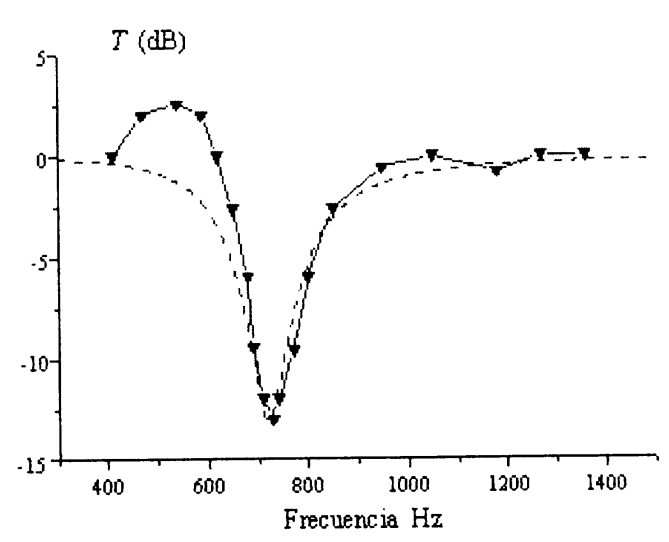

Figura 9. Coeficiente de transmisión en función de la frecuencia cuando un RH de frecuencia $f_{H}=720 \pm 10 \mathrm{~Hz}$ se inserta en tubo "infinito". 


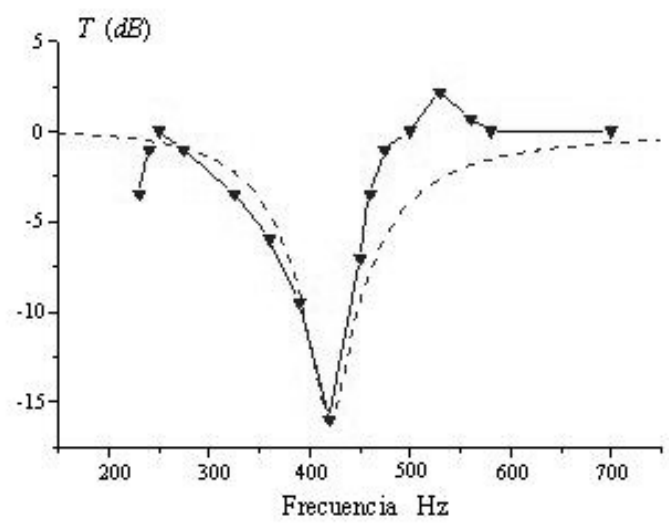

Figura 10. Idem que figura 9 para un resonador de $420 \pm 10 \mathrm{~Hz}$.

\section{Conclusiones}

Cuando se utiliza un $R H$ como filtro de ondas estacionarias su propósito, en general, es el de eliminar uno o varios de los modos resonantes del recinto en el cual se lo inserta. Este objetivo se logra si la frecuencia $f_{H}$ del $R H$ coincide con la frecuencia del modo que se desea eliminar. El filtro es más efectivo cuando mayor es su resistencia. Si la resistencia del filtro es muy pequeña se elimina el modo cuya frecuencia está próxima a $f_{H}$, pero aparecen dos modos adicionales con amplitudes que dependen fuertemente de la resistencia acústica del resonador $r_{H}$. De acuerdo con nuestros resultados teóricos, confirmados por las mediciones, los niveles de amplitud de estos modos se mantienen relativamente pequeños si $r_{H} \geq 0.1$. En esta situación el $R H$ no solamente elimina el modo que está próximo a su frecuencia de resonancia sino que también reduce la amplitud de los modos vecinos, como se observa en las figuras 3 y 5 .

Si en el tubo se tienen ondas propagantes, el efecto de filtrado del $R H$ se describe mediante el coeficiente de transmisión. El coeficiente de transmisión que medimos experimentalmente, después de tener la precaución de insertar una terminación absorbente, concuerda razonablemente bien con la curva teórica como se observa en las figuras (9) y (10). La forma de estas curvas depende de los parámetros $r_{H}$ y $\varkappa$ del RH. El mínimo valor de $T$ es igual a $T_{\text {min }}=4 r_{H}^{2}$ (que es menor cuando más pequeño es $r_{H}$ ) y el ancho de banda en frecuencia de la misma es $\Delta f_{1 / 2}=f_{H} / 2 \varkappa$. Observemos que el parámetro $r_{H}$ juega un papel principal, aunque disímil, según se use el $R H$ para "filtrar" ondas estacionarias o propagantes

En conclusión, la capacidad de un dado $R H$ para filtrar ondas estacionarias y ondas propagantes es diferente y los modelos teóricos que dan cuenta de este comportamiento son, por lo tanto, distintos. En particular, un excelente filtro para ondas propagantes que se obtiene cuando $r_{H} \rightarrow 0$, puede tener un mal comportamiento como filtro de ondas estacionarias.

En la Facultad de Ciencias e Ingeniería de Rosario, se incorporó el estudio experimental de filtros acústicos, con una explicación teórica cualitativa, en el curso de Física de las Ondas del segundo año, y con el nivel presentado en este trabajo para los alumnos del tercer año de las carreras de Ingeniería Mecánica y Electrónica.

\section{Referencias}

Cameron, T. y Russell D., Laboratory Instruction in Acoustic and Vibration $\diamond$, Proceedings of the 1996 ASEE, Annual Conference and Exposition,, Washington, D.C., Junio 23 - 26, (1996).

Brewer, R., The Physics of Acoustics, Thesis, Reed College, Hawai University, (1996).

Kinsler, L. E., Frey A. R., Coppens A. B. y J. V. Sanders, Fundamentals of Acoustics, tercera edición John Wiley \& Sons, (1982).

Russell, D. A. Acoustic high-pass, low-pass and bandstop filters. Este trabajo está disponible en la Web, en la dirección: http://www.kettering.edu/ drussell/GMIAcoustics/Filters-Frame.html, (1996)

Tipler, P. A., Física, tercera edición, Editorial Reverté S. A., (1995).

\section{Anexo 1. El resonador Helmholtz}

El RH consiste de una cavidad de paredes rígidas de volumen $V$ con un cuello de área $S_{H}$ y longitud $l_{H}$ (ver figura 11). Si la longitud de onda $\lambda \gg l_{H}$, el fluido en el cuello se mueve como una unidad y se comporta como el elemento masa de un oscilador a resorte. Si $\lambda \gg V^{1 / 3}$, la presión acústica dentro de la cavidad proporciona el elemento elástico de este oscilador.

Vamos a analizar al resonador de Helmholtz calculando los valores del sistema mecánico análogo. El fluido en el cuello tiene una masa efectiva total

$$
m=\rho_{0} S_{H} l_{H}
$$

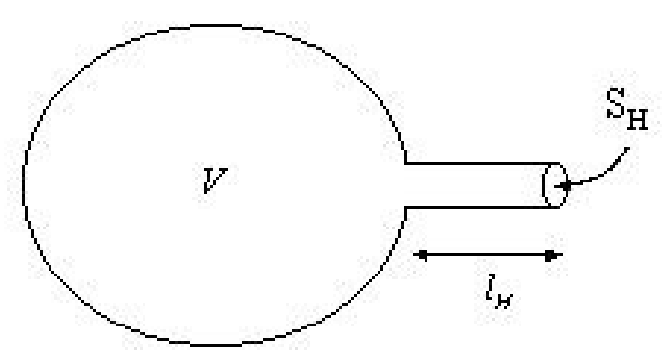

Figura 11. Un Resonador Helmholtz.

Si la masa del cuello se desplaza hacia fuera una distancia $x$ el volumen de la cavidad cambia en $\Delta V$. Si suponemos que el proceso es adiabático, el incremento de la presión $\Delta p$ se relaciona con la variación de volumen $\Delta V$, a través de la relación:

$$
\Delta p=-B \frac{\Delta V}{V}
$$


donde $B=\rho c^{2}$ es el módulo de compresibilidad adiabático del gas y c es la velocidad del sonido (Tipler, 1995). Como $\Delta V=-S_{H} x$ obtenemos que la fuerza restauradora que actúa sobre la masa $m$ es:

$$
F_{x}=-B \frac{S_{H}^{2}}{V} x
$$

La "constante elástica" del oscilador es, por lo tanto,

$$
s=\rho_{0} c^{2} \frac{S_{H}}{V}
$$

Vamos a designar con $R_{m}$ la resistencia del resonador que tiene en cuenta tanto las pérdidas por radiación de sonido como a las pérdidas viscosas en el cuello. Es muy difícil encontrar una expresión analítica para esta resistencia.

Si una onda sonora de frecuencia $\omega$ y de amplitud $p$, incide sobre el cuello del resonador se produce una fuerza excitadora instantánea $F_{e}$ que viene dada por

$$
F_{x}=p S_{H} e^{i \omega t}
$$

La ecuación diferencial para el desplazamiento $x$ del fluido en el interior del cuello del resonador es

$$
m \frac{d^{2} x}{d t^{2}}+R_{m} \frac{d x}{d t}+s x=F_{e}=p S_{H} e^{i \omega t}
$$

Puesto que la ecuación es idéntica a la de un oscilador forzado, su solución puede obtenerse por analogía.En particular la impedancia mecánica del $R H$ es

$Z_{m}=\frac{F_{e}}{u}=\frac{p S_{H} e^{i \omega t}}{d x / d t}=R_{m}+i\left(\omega m-\frac{s}{\omega}\right)=R_{m}+i X_{m}$

donde $u=d x / d t$ es la velocidad de un elemento de fluido.

La frecuencia de resonancia tiene lugar cuando la reactancia $X_{m}$ es cero, esto es, cuando:

$$
\omega_{H}=\sqrt{\frac{s}{m}}=c \sqrt{\frac{S_{H}}{l_{H} V}}
$$

En términos de $\omega_{H}$ la reactancia mecánica del oscilador es:

$$
X_{m}=m \omega_{H}\left(\frac{\omega}{\omega_{H}}-\frac{\omega_{H}}{\omega}\right)
$$

La impedancia acústica del resonador $Z_{H}$ está relacionada con su impedancia mecánica a través de la relación, $Z_{H}=p / U=\left(F_{e} / S_{H}\right) /\left(u S_{H}\right)=Z_{m} / S_{H}^{2}$, esto es:

$$
Z_{H}=\frac{Z_{m}}{S_{H}^{2}}
$$

Reemplazando $R_{m}$ y $X_{m}$ en esta ecuación, obtenemos la siguiente expresión para la impedancia acústica $Z_{H}$ del $R H$ :

$$
Z_{H}=\frac{R_{m}}{S_{H}^{2}}+i \frac{\rho_{0} l_{H} \omega_{H}}{S_{H}}\left(\frac{\omega}{\omega_{H}}-\frac{\omega_{H}}{\omega}\right)
$$

La reactancia $X_{H}$ del resonador, de acuerdo a la ecuación (A11), viene dada por

$$
X_{H}=\frac{\rho_{0} l_{H} \omega_{H}}{S_{H}}\left(\frac{\omega}{\omega_{H}}-\frac{\omega_{H}}{\omega}\right)
$$

La impedancia acústica del tubo dondew se inserta el RH es

$$
Z_{0}=\frac{\rho_{0} c}{S_{0}}
$$

La reactancia "normalizada" del RH es, por lo tanto

$$
r_{H}=\frac{X_{H}}{Z_{0}}=\varkappa\left(\frac{f}{f_{H}}-\frac{f_{H}}{f}\right)
$$

donde

$$
\varkappa=\frac{\rho_{0} l_{H} \omega_{H} / S_{H}}{\rho_{0} c / S_{0}}=2 \pi \frac{l_{H} f_{H}}{c} \frac{S_{0}}{S_{H}}
$$

\section{Anexo 2. Descripción del equipo experimental}

En la realización de este trabajo se utilizaron los siguientes elementos:

1.Un tubo de $P V C$ de $1 m$ de longitud y $2,7 \mathrm{~cm}$ de diámetro. A este tubo se le han practicado tres orificios, a $0,25 \mathrm{~cm}, 0,50 \mathrm{~cm}$ y $0,75 \mathrm{~cm}$ de uno de sus extremos, respectivamente para insertar el resonador Helmholtz y los micrófonos.

2. Un parlante y un micrófono con buena respuesta en frecuencia.

3. Una oscilador de audio de amplitud y frecuencia variable.

4. Un amplificador de audio y un osciloscopio.

5. Se construyeron varios $R H$. En este trabajo mencionamos a tres de ellos, a los que identificaremos como resonadores $A, B$ y $C$ respectivamente.

Parámetros de los $\mathrm{RH}$
utilizados
$l_{H}$
$S_{H}$
$V$
$f_{H}$
$\varkappa$

